\title{
Atributos Edáficos em Áreas de Agricultura, Pastagem e Três Estágios Sucessionais de Floresta
}

\author{
Fernando Silva Coutinho ${ }^{1}$, Marcos Gervasio Pereira ${ }^{2}$, \\ Carlos Eduardo Gabriel Menezes ${ }^{3}$, Roni Fernandes Guareschi², \\ Shirlei Almeida Assunção ${ }^{2}$ \\ ${ }^{1}$ Pessoas, Inovação e Performance - PRODAP, Belo Horizonte/MG, Brasil \\ ${ }^{2}$ Departamento de Solos, Universidade Federal Rural do Rio de Janeiro - UFRRJ, Seropédica/RJ, Brasil \\ ${ }^{3}$ Instituto Federal do Rio de Janeiro - IFRJ, Rio de Janeiro/RJ, Brasil
}

\begin{abstract}
RESUMO
O objetivo deste trabalho foi avaliar os teores de carbono orgânico total e alguns atributos físicos (densidade do solo, macroporosidade, microporosidade, diâmetro médio ponderado e geométrico de agregados) de um Cambissolo Háplico sob fragmentos florestais de Mata Atlântica em estágio inicial; intermediário e avançado, e compará-los à áreas de pastagem e agricultura (anual e perene). A regeneração de florestas secundárias em função do estágio de recuperação contribuiu para o aumento de carbono orgânico total e da macroporosidade, reduzindo os valores de densidade do solo, diâmetro médio ponderado e geométrico de agregados. Na área de pastagem foram verificados valores de carbono orgânico total, densidade do solo, macroporosidade, microporosidade e diâmetro médio ponderado e geométrico de agregados similares aos observados nas áreas de floresta. Na área de agricultura anual observou-se redução dos teores de carbono orgânico total, macroporosidade, diâmetro médio ponderado e geométrico de agregados e aumento da densidade do solo em função do manejo adotado.
\end{abstract}

Palavras-chave: fertilidade do solo, física do solo.

\section{Attributes of Soil Under Agriculture, Grazing and Three Succession Stages of Forest}

\begin{abstract}
This study aimed to evaluate changes in the content of total organic carbon (TOC) and some physical properties (Bulk density, macroporosity, microporosity, mean weight diameter and mean geometric diameter of aggregates) of a Inceptsol in forest fragments of the Atlantic Forest in early; intermediate and advanced stage, and compare them to pasture and agriculture (annual and perennial) areas. Secondary forests regeneration due to the recovery stage contributed to the increase of total organic carbon and macroporosity, reducing soil density values, and weighted geometric mean diameter of aggregates. In the pasture area Values of total organic carbon values, soil bulk density, macroporosity, microporosity and weighted mean and geometric diameters of aggregates were similar to those observed in forest areas. In the annual agriculture area we observed reduction of total organic carbon, macroporosity, and weighted mean and geometric diameter of aggregates and increased soil density according to the adopted management.
\end{abstract}

Keywords: soil fertility, soil physics. 


\section{INTRODUÇÃO}

A ocupação da sub-bacia do Ribeirão Cachimbal não considerou a aptidão das terras e a necessidade de uma política voltada à definição de seu uso adequado, intensificando os processos de degradação ambiental já existentes (Menezes, 2008; Silva et al., 2012). Essa região apresenta pequenas propriedades com agricultura predominantemente de subsistência, caracterizada pelo policultivo nas encostas, com práticas de manejo que aceleram a degradação do solo (Menezes, 2008; Silva et al., 2012).

O desmatamento para o cultivo agrícola em regiões de relevo movimentado, ou seja, que apresentam vales profundos e estreitos dissecados, pode originar uma paisagem com diferentes graus de degradação, sendo que os principiais indicadores desse processo são a diminuição na capacidade de retenção de água no solo, baixa estabilidade do solo, favorecendo o aumento do processo erosivo, e as reduções nos níveis de fertilidade e na capacidade de o solo estocar carbono - C (Machado et al., 2010; Tabarelli et al., 2009; Macedo et al., 2008; Boddey et al., 2006).

No entanto, Balbinot (2009) menciona que as sucessões vegetais de áreas de floresta sob pastagem (PA) promovem alterações na quantidade de biomassa estocada em cada fase de regeneração florestal, favorecendo a melhoria dos atributos físicos e químicos do solo por meio da adição de matéria orgânica. Em consonância, Schedlbauer \& Kavanagh (2008) mencionam que reduções nas perdas de carbono do solo decorrentes da conversão da floresta tropical em áreas de pastagem podem muitas vezes ser restauradas por meio do desenvolvimento de florestas secundárias. Tal padrão é constatado nos trabalhos de Silva et al. (2012), Bochner (2007), Menezes (2008), Loss et al. (2006) e Fontana et al. (2005), no qual a regeneração de florestas secundárias aumentaram o teor de $\mathrm{C}$ no solo, quando comparado ao de áreas adjacentes de pastagem, agricultura anual e/ou perene.

$\mathrm{Na}$ literatura encontram-se alguns estudos em que têm sido observados maiores valores de densidade do solo (Ds) e menores valores de volume total de poros (VTP), diâmetro médio ponderado (DMP) e diâmetro médio geométrico (DMG) de agregados em áreas de pastagem (Santos et al., 2010; Melloni et al., 2008; Bochner, 2007) e agricultura (Bochner, 2007; Sá et al.,
2003) em comparação com a floresta secundária. Tais resultados são justificados pela compactação do solo, pelo pisoteio dos animais na área de pastagem; aração e gradagem nas áreas de agricultura e pelo maior e constante acúmulo de resíduos vegetais na superfície do solo (RVS) nas áreas de floresta em relação aos sistemas manejados. No entanto, os estudos de Coutinho (2012), Oliveira et al. (2008) e Barreto et al. (2006) não observaram diferença significativa de Ds e VTP para essas coberturas. Esse padrão pode decorrer ao pequeno tempo de sucessão da floresta secundária, ou seja, a regeneração da floresta ainda não foi capaz de reduzir os impactos na Ds causados pela pastagem que a antecedeu (Oliveira et al., 2008).

Diante do exposto, o objetivo deste trabalho foi avaliar alterações no teor de carbono orgânico total (COT) e em alguns atributos físicos do solo em áreas de fragmentos florestais da Mata Atlântica com diferentes estágios de regeneração, e compará-las às de áreas de pastagem e agricultura (anual e perene), no município de Pinheiral, RJ.

\section{MATERIAL E MÉTODOS}

O estudo foi realizado no município de Pinheiral, região do Médio Vale do Paraíba do Sul, na sub-bacia do ribeirão Cachimbal, que compõe a bacia hidrográfica do rio Paraíba do Sul, localizado entre as latitudes $22^{\circ} 33^{\prime} \mathrm{S}$ e $22^{\circ} 38^{\prime} \mathrm{S}$ e entre as longitudes $43^{\circ} 57^{\prime} \mathrm{W}$ e $44^{\circ} 5^{\prime} \mathrm{W}$ (Figura 1).

O clima da região é classificado como temperado de inverno seco e verão chuvoso (Köppen, 1948). Precipitação e temperatura médias anuais são $1.300 \mathrm{~mm}$ e $21^{\circ} \mathrm{C}$, respectivamente.

A região está inserida no domínio ecológico da Mata Atlântica, cuja vegetação original denomina-se Floresta Estacional Semidecidual Submontana (IBGE, 2012), característica de zonas de altitudes entre 300 e $800 \mathrm{~m}$. A cobertura vegetal atualmente dominante na região é constituída por pastagens manejadas e espontâneas não manejadas, que se apresentam em diferentes estágios de degradação, nível de uso e/ou abandono.

Os solos identificados nas seis unidades de estudo foram classificados como Cambissolos Háplicos Tb Distrófico típico (EMBRAPA, 2006), uma das classes predominantes nas cotas mais altas da sub-bacia 


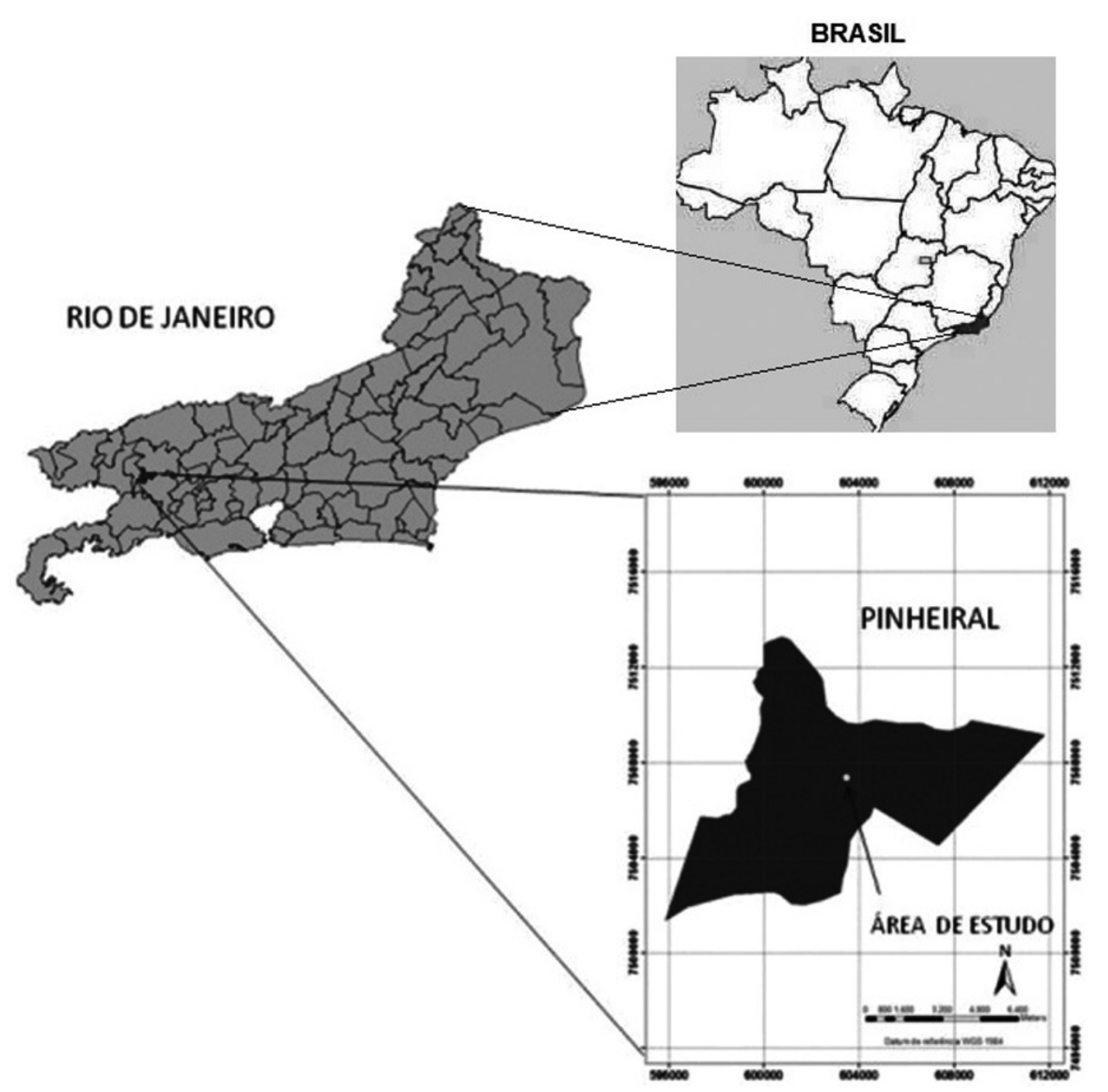

Figura 1. Localização geográfica das áreas de estudo. Fonte: adaptado de Mateus et al. (2013). Figure 1. Geographic location of the study areas. Source: Adapted from Mateus et al. (2013).

(Coutinho, 2012). Todas as áreas estão localizadas na posição de terço superior de encosta.

Para o estudo foram selecionadas 6 áreas, sendo 3 delas compostas por florestas secundárias, que compõem um único fragmento florestal. As florestas encontram-se em diferentes estágios de sucessão, a saber: fragmento em estágio inicial (FIN); fragmento em estágio intermediário (FIT); e fragmento em estágio avançado (FAV). As tipologias florestais foram classificadas de acordo com a resolução do Conselho Nacional do Meio Ambiente - Conama (006/1994). Também foram utilizadas no estudo uma área de pastagem manejada com Brachiaria decumbens e Paspalum notatum (PA), uma área de agricultura perene cultivada com citros (AP) e uma área de agricultura anual cultivada alternadamente com milho, feijão e mandioca (AA).

A área FIN apresenta cobertura florestal pouco densa, característica de estágio inicial de sucessão (Brasil, 1994), originada por utilização da área até 1985 como pastagem espontânea, manejada por roçadas anuais e queimadas eventuais, quando foi ocupada por pequenos agricultores que apenas a cercaram, permitindo assim a regeneração florestal.

A área FIT apresenta-se contígua e de mesma cota que a FIN e até 1985 se encontrava sob cobertura de pasto espontâneo com formação inicial de capoeira, sendo mantida protegida até os dias atuais, o que 
permitiu o desenvolvimento sucessional típico do estágio médio (Brasil, 1994), caracterizado por uma cobertura arbustivo/arbórea mais fechada que a do estágio inicial.

A área FAV apresenta uma cobertura florestal densa e mais bem estruturada do que as anteriores, o que permite enquadrá-la nesse estágio sucessional (Brasil, 1994) - a sucessão teve início após a decadência da cafeicultura na região e constitui o fragmento mais antigo da porção inferior da sub-bacia.

Entre os sistemas manejados, a área de PA foi explorada com pastagem espontânea desde a década de 1950. A partir da década de 1990 foi implantada Brachiaria decumbens e, a partir daí, a pastagem foi mantida por meio de roçadas anuais. Com a prática de queima coibida, com o decorrer dos anos ressurgiu nessa paisagem a gramínea conhecida como grama-batatais (Paspalum notatum), que passou a coexistir com a braquiária introduzida. Já a área de AP foi utilizada como pastagem até a década de 1990 . Após esse período, voltou a ser usada para atividades agrícolas, com a finalidade de subsistência (milho e feijão) e atualmente encontra-se com cultivo de citros em sistema de preparo convencional (sem adubação e sem calagem), com capinas manuais periódicas. Por fim, a área de AA vem sendo utilizada para agricultura de subsistência (milho, feijão, mandioca) há aproximadamente 20 anos. No momento da coleta das amostras de terra para o estudo, essa área encontrava-se com a cultura de mandioca. O sistema de preparo é o convencional (sem adubação e sem calagem), com capinas manuais periódicas.

Em cada área de estudo foi demarcada uma gleba representativa de 0,04 ha $(20 \times 20 \mathrm{~m})$, e em cada uma delas foram abertas cinco trincheiras de aproximadamente $1 \times 1 \mathrm{~m}$ de superfície e $1 \mathrm{~m}$ de profundidade em posição aleatória. $\mathrm{O}$ delineamento utilizado foi inteiramente casualizado, com cinco repetições para cada área e profundidade. Em cada uma das trincheiras, nas diferentes áreas, foi realizada a coleta de amostras indeformadas, com auxílio de um anel volumétrico (EMBRAPA, 1997), nas profundidades 0,0-0,05; $0,05-0,1 ; 0,1-0,2 ; 0,2-0,4 \mathrm{~m}$. Nessas profundidades também foram coletadas amostras deformadas, que foram secas ao ar, destorroadas e passadas por peneira de $2 \mathrm{~mm}$ de malha, obtendo-se a terra fina seca ao ar na qual foi realizada a maioria das análises, inclusive caracterização química e análise granulométrica (EMBRAPA, 1997) (Tabela 1).

A determinação do $\mathrm{C}$ orgânico total (COT) foi realizada pelo método de oxidação via úmida, com aquecimento externo, segundo a metodologia proposta por Yeomans \& Bremner (1988).

A densidade do solo (Ds) foi determinada em amostras indeformadas coletadas com amostrador tipo Uhland, com anéis volumétricos de 0,05 $\mathrm{m}$ de altura e 0,05 $\mathrm{m}$ de diâmetro ao longo das camadas avaliadas. Após a coleta, o material foi seco em estufa $\left(105^{\circ} \mathrm{C}\right)$ até a obtenção de massa constante. Posteriormente, a densidade foi calculada pela seguinte Equação 1:

Ds $\left(\mathrm{Mg} \mathrm{m}^{-3}\right)=\mathrm{Ms} / \mathrm{Vs}$

em que: $\mathrm{Ms}=$ massa do solo em mg; Vs = volume do solo em $\mathrm{m}^{-3}$.

Em amostras indeformadas coletadas com amostrador tipo Uhland, com anéis de PVC, também foram realizadas as determinações de macro e microporosidade, utilizando-se o método da mesa de tensão com coluna d'água de 0,60 m (EMBRAPA, 1997).

Para avaliação da estabilidade dos agregados foram coletadas amostras indeformadas nas profundidades 0,0-0,05 e 0,05-0,1 m do perfil do solo. Após essa etapa, as amostras foram secas ao ar e, em seguida, passadas por peneiras de 8 e $4 \mathrm{~mm}$ de malha (EMBRAPA, 1997). Em seguida, foram pesadas $25 \mathrm{~g}$ das amostras que ficaram retidas na peneira de $4 \mathrm{~mm}$ e, depois de umedecidas com pulverizador, colocadas em um jogo de peneiras de malhas 2,00; 1,00;0,50;0,25 e 0,105 mm, sendo submetidas à agitação vertical no aparelho Yooder, durante $15 \mathrm{~min}$. O material retido em cada peneira foi colocado em placas previamente pesadas e identificadas e levado à estufa a $65^{\circ} \mathrm{C}$ até peso constante. Após a secagem, quantificou-se a massa dos agregados retidos em cada peneira. Com esses dados de massa foram calculados o diâmetro médio ponderado (DMP) e o diâmetro médio geométrico dos agregados, utilizando equações, conforme Castro et al. (1998).

Para todos os dados, em cada profundidade, foi feita a avaliação da normalidade dos dados (Lilliefors), homogeneidade das variâncias dos erros pelo Teste de Cochran e Barttlet. Posteriormente, os resultados foram submetidos a análise de variância com aplicação do teste $\mathrm{F}$ e os valores médios comparados entre si 
Tabela 1. Atributos químicos e análise granulométrica das áreas de estudo.

Table 1. Chemical properties and particle size analysis of the study areas.

\begin{tabular}{|c|c|c|c|c|c|c|c|c|c|c|}
\hline \multirow{2}{*}{ Áreas $^{1}$} & \multirow{2}{*}{$\begin{array}{c}\mathrm{pH} \\
\mathrm{H}_{2} \mathrm{O}\end{array}$} & \multirow{2}{*}{$\underset{\mathrm{mg} \mathrm{kg}^{-1}}{\mathbf{P}}$} & $\mathbf{C a}$ & Mg & $\mathbf{K}$ & Al & $\mathbf{H}+\mathbf{A l}$ & \multicolumn{3}{|c|}{ Granulometria $\left(\mathrm{g} \mathrm{kg}^{-1}\right)$} \\
\hline & & & \multicolumn{5}{|c|}{---------- $\mathrm{cmol}_{\mathrm{c}} \mathrm{kg}^{-1}$----------- } & Argila & Areia & Silte \\
\hline \multicolumn{11}{|c|}{ 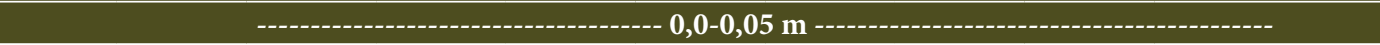 } \\
\hline FIN & $4,8^{*}$ & 2 & 1,6 & 1,4 & 0,09 & 0,0 & 10,3 & 330 & 490 & 180 \\
\hline FIT & 5,6 & 2 & 4,9 & 5,0 & 0,31 & 0,1 & 7,4 & 290 & 540 & 170 \\
\hline FAV & 3,9 & 2 & 2,2 & 1,5 & 0,16 & 0,4 & 8,9 & 240 & 650 & 110 \\
\hline PA & 5,6 & 3 & 5,0 & 4,9 & 0,07 & 0,0 & 5,5 & 260 & 550 & 190 \\
\hline $\mathrm{AP}$ & 5,7 & 3 & 2,2 & 2,7 & 0,07 & 0,2 & 6,1 & 230 & 620 & 150 \\
\hline AA & 5,8 & 2 & 2,4 & 3,6 & 0,19 & 0,2 & 4,3 & 230 & 630 & 140 \\
\hline \multicolumn{11}{|c|}{ - } \\
\hline FIN & 4,5 & 2 & 0,5 & 1,3 & 0,07 & 0,0 & 7,9 & 300 & 540 & 160 \\
\hline FIT & 4,7 & 2 & 2,7 & 3,0 & 0,19 & 0,4 & 7,3 & 300 & 500 & 200 \\
\hline FAV & 3,8 & 2 & 1,3 & 1,4 & 0,14 & 0,8 & 7,9 & 300 & 560 & 140 \\
\hline PA & 5,1 & 2 & 2,0 & 5,3 & 0,04 & 0,0 & 5,5 & 270 & 560 & 170 \\
\hline AP & 5,4 & 2 & 1,8 & 2,6 & 0,04 & 0,6 & 5,4 & 270 & 580 & 150 \\
\hline AA & 5,7 & 0 & 2,3 & 2,6 & 0,05 & 0,6 & 5,4 & 290 & 550 & 160 \\
\hline \multicolumn{11}{|c|}{ 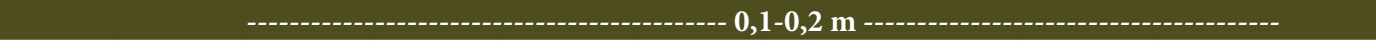 } \\
\hline FIN & 4,5 & 0 & 0,3 & 0,6 & 0,03 & 0,0 & 7,4 & 340 & 490 & 170 \\
\hline FIT & 4,5 & 1 & 1,5 & 1,7 & 0,12 & 0,8 & 6,0 & 310 & 500 & 190 \\
\hline FAV & 3,8 & 1 & 1,3 & 1,7 & 0,09 & 0,8 & 6,6 & 340 & 520 & 140 \\
\hline PA & 6,0 & 1 & 1,2 & 1,9 & 0,03 & 0,0 & 4,6 & 290 & 540 & 170 \\
\hline $\mathrm{AP}$ & 5,3 & 0 & 1,6 & 1,9 & 0,03 & 0,5 & 5,2 & 310 & 570 & 120 \\
\hline AA & 5,5 & 0 & 1,5 & 3,0 & 0,04 & 0,6 & 4,6 & 310 & 580 & 110 \\
\hline \multicolumn{11}{|c|}{ 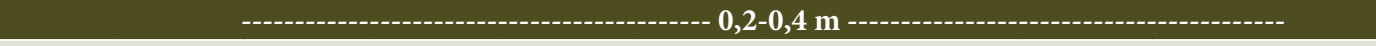 } \\
\hline FIN & 4,4 & 0 & 0,3 & 0,7 & 0,02 & 0,1 & 7,6 & 340 & 470 & 190 \\
\hline FIT & 4,4 & 0 & 0,9 & 1,7 & 0,07 & 0,9 & 7,2 & 340 & 450 & 210 \\
\hline FAV & 3,9 & 1 & 1,1 & 1,1 & 0,07 & 0,7 & 5,8 & 330 & 500 & 170 \\
\hline PA & 5,2 & 1 & 1,4 & 1,5 & 0,02 & 0,1 & 3,9 & 320 & 550 & 130 \\
\hline AP & 5,1 & 0 & 1,5 & 2,7 & 0,03 & 0,3 & 4,4 & 320 & 540 & 140 \\
\hline AA & 5,4 & 0 & 1,5 & 2,6 & 0,03 & 0,5 & 5,1 & 290 & 590 & 120 \\
\hline
\end{tabular}

${ }^{*}$ Média de cinco repetições, sendo que valores com a mesma letra na coluna não diferem entre si pelo teste de Bonferroni a $5 \%$. ${ }^{1} \mathrm{FIN}$ - Fragmento de floresta secundária em estágio inicial; FIT - Fragmento de floresta secundária em estágio intermediário; FAV - Fragmento de floresta secundária em estágio avançado; PA - Pastagem; AP - Agricultura perene; AA - Agricultura anual.

pelo Teste Scott-Knott, com a utilização do programa estatístico SAEG versão 5.0.

Realizou-se também a análise de componentes principais (ACP), por meio de planilhas eletrônicas. A ACP é utilizada para reduzir as dimensões dos dados e, consequentemente, facilitar a análise.

\section{RESULTADOS E DISCUSSÃO}

Na Figura 2 verifica-se que a área de fragmento de floresta secundária em estágio avançado (FAV) foi a que apresentou maior teor de carbono em comparação às áreas de fragmento de floresta secundária em estágio inicial (FIN) (0,0-0,4 m) e fragmento de floresta secundária em estágio intermediário (FIT) (0,0-0,1 e 0,2-0,4 m), bem como em relação às áreas de pastagem (PA) (0,05-0,1 e 0,1-0,2 m) e agricultura perene $(\mathrm{AP})$ e anual $(\mathrm{AA})(0,0-0,4 \mathrm{~m})$. Esse padrão ocorre devido a fragmento de floresta secundária em estágio avançado (FAV) ser um sistema sem pertubação antrópica, com maior diversidade de vegetais e com aporte constante de resíduos vegetais na superfície do solo. Padrões similares ao verificado neste estudo foram observados por Silva et al. (2012), Bochner (2007), Menezes (2008), Fontana et al. (2005) e Loss et al. (2006). Os autores verificaram que a regeneração de florestas secundárias contribui para o aumento do teor de $\mathrm{C}$ no solo, quando comparadas a áreas adjacentes de pastagem, agricultura anual e/ou perene. 


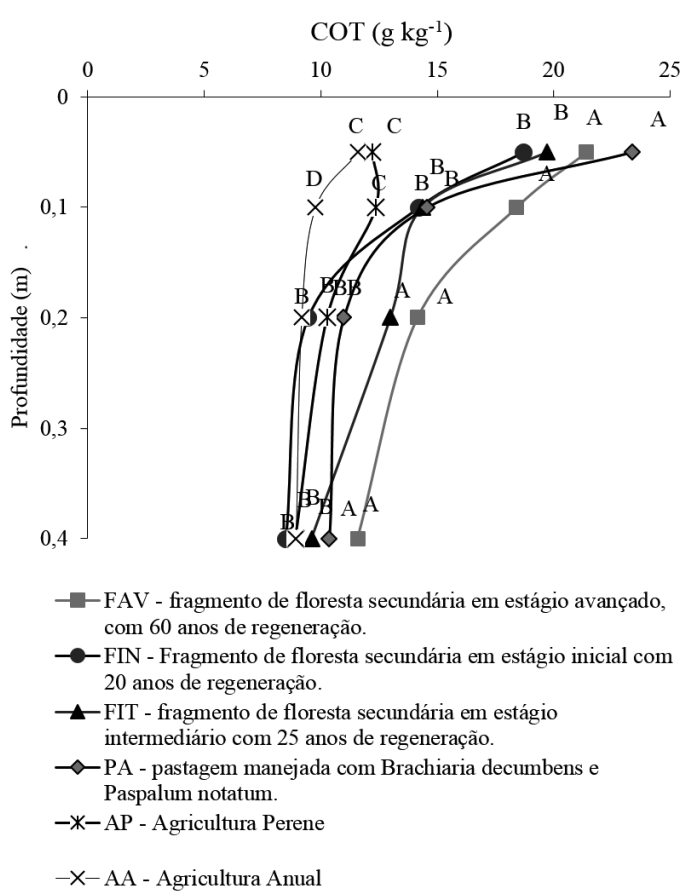

Figura 2. Valores médios de carbono orgânico total (COT) nas áreas avaliadas. Letras maiúsculas comparam os tratamentos dentro de uma mesma profundidade, através do Teste Scott-Knott $(\mathrm{P} \leq 0,05)$.

Figure 2. Mean total organic carbon (COT) in the evaluated areas. Uppercase comparing treatments within the same depth, by testing Scott-Knott $(\mathrm{P} \leq 0.05)$.

Os solos sob as áreas de fragmento de floresta secundária em estágio inicial (FIN) (0,0-0,10 m) e fragmento de floresta secundária em estágio intermediário (FIT) (0,0-0,20 m) também apresentaram maior teor de carbono em comparação às áreas de agricultura perene (AP) e agricultura anual (AA) (Figura 2). Esse padrão deve-se ao maior revolvimento das camadas superficiais das áreas de agricultura, que ocasionam a ruptura dos agregados, aumentando a exposição de novas superfícies ao ataque microbiano, além do aumento da pressão parcial de oxigênio, que acarreta perdas de carbono orgânico total (COT) por oxidação (Bochner, 2007). Além disso, pode-se inferir que a falta de adubação e calagem das áreas de agricultura gera uma baixa adição de resíduos vegetais ao solo (constatação visual nas áreas) quando comparadas às áreas de FIN e FIT, contribuindo dessa forma para um menor teor de carbono orgânico no solo. Resultados semelhantes foram observados por Fontana et al. (2011), que também constataram maior teor de carbono em áreas de floresta secundária em comparação a áreas de agricultura. Os autores também atribuem este resultado à baixa adição de resíduos pela agricultura, bem como pelo manejo convencional adotado na área.

Ressalta-se também, à semelhança, a superioridade do teor de carbono (0,0-0,05 e 0,2-0,4 m) entre as áreas de pastagem (PA) e fragmento de floresta secundária em estágio avançado (FAV) em comparação às demais áreas (Figura 2). Esse resultado pode decorrer do fato de na área PA não ser realizado o revolvimento do solo e devido ao sistema radicular das gramíneas explorar um maior volume de solo, contribuindo com uma maior adição de COT tanto nas camadas superficiais quanto em subsuperfície. De acordo com Silva et al. (2012), a semelhança no teor de carbono de áreas florestais e de pastagem pode estar associada à maior quantidade de resíduos vegetais produzidos pelas espécies florestais, bem como à eficiente ciclagem de nutrientes que ocorre em ambas as áreas.

Até $0,1 \mathrm{~m}$ de profundidade do solo, as áreas de agricultura (perene e anual) apresentaram maior valor de densidade do solo (Ds) em comparação com as demais áreas (Tabela 2). No entanto, verifica-se que na profundidade de 0,2-0,4 m, as áreas de fragmento de floresta secundária em estágio avançado (FAV) e pastagem (PA) foram as que apresentaram o menor valor de Ds (Tabela 2). Através desses resultados observa-se a influência do carbono orgânico do solo sobre a Ds, ou seja, as áreas de floresta secundária e pastagem que apresentaram maior valor de carbono orgânico que as áreas de agricultura foram aquelas nas quais se quantificou os menores valores de Ds. Bochner (2007) menciona que os baixos valores de densidade do solo verificados em áreas florestais devem-se, em parte, à espessa camada de serapilheira e à grande presença de raízes finas que promovem elevação no teor de matéria orgânica, melhoria na agregação e consequente diminuição da densidade. Resultados semelhantes foram encontrados por Silva et al. (2006), que ao avaliarem áreas de agricultura e floresta secundária, constataram maior valor de densidade na área de agricultura (plantio de mandioca). Os autores também associaram tais resultados ao menor teor de matéria orgânica da área de agricultura, bem como à maior ação antrópica.

Observa-se um aumento dos valores de macroporosidade $(0,0-0,4 \mathrm{~m})$ em função do tempo 
Tabela 2. Densidade do solo (Ds), macro (Ma) e microporosidade (Mi) e diâmetro médio ponderado (DMP) e geométrico (DMG) de agregados das áreas de estudo.

Table 2. Soil density (Ds), macro (Ma) and microporosity (Mi) and mean diameter weight (MDW) and geometric (MDG) of aggregates of the study areas.

\begin{tabular}{|c|c|c|c|c|c|c|}
\hline \multirow{3}{*}{$\begin{array}{l}\text { Profundidade } \\
\text { (m) }\end{array}$} & \multicolumn{6}{|c|}{ Áreas de estudo } \\
\hline & FIN $^{1}$ & FIT & FAV & PA & $\mathbf{A P}$ & AA \\
\hline & \multicolumn{6}{|c|}{ Ds $\left(\mathrm{Mg} \mathrm{m}^{-3}\right)$} \\
\hline $0-0,05$ & $1,22 \mathrm{~B}^{\star}$ & $1,23 \mathrm{~B}$ & $1,13 \mathrm{~B}$ & $1,06 \mathrm{~B}$ & $1,30 \mathrm{~A}$ & $1,31 \mathrm{~A}$ \\
\hline $0,05-0,1$ & $1,16 \mathrm{~B}$ & $1,14 \mathrm{~B}$ & $0,96 \mathrm{~B}$ & $1,08 \mathrm{~B}$ & $1,24 \mathrm{~A}$ & $1,20 \mathrm{~A}$ \\
\hline $0,1-0,2$ & $1,33 \mathrm{~A}$ & $1,18 \mathrm{~A}$ & $1,08 \mathrm{~A}$ & $1,28 \mathrm{~A}$ & $1,36 \mathrm{~A}$ & $1,22 \mathrm{~A}$ \\
\hline \multirow[t]{2}{*}{$0,2-0,4$} & $1,35 \mathrm{~A}$ & $1,30 \mathrm{~A}$ & $1,14 \mathrm{~B}$ & $1,05 \mathrm{~B}$ & $1,27 \mathrm{~A}$ & $1,34 \mathrm{~A}$ \\
\hline & \multicolumn{6}{|c|}{$\operatorname{Ma}\left(\mathbf{m}^{3} \mathbf{m}^{3}\right)$} \\
\hline $0-0,05$ & $0,9 \mathrm{~B}$ & $1,3 \mathrm{~B}$ & $1,5 \mathrm{~A}$ & $1,6 \mathrm{~A}$ & $1,8 \mathrm{~A}$ & $1,7 \mathrm{~A}$ \\
\hline $0,05-0,1$ & $1,1 \mathrm{~B}$ & $0,3 \mathrm{~B}$ & $1,7 \mathrm{~A}$ & $1,5 \mathrm{~A}$ & $2,0 \mathrm{~A}$ & $1,7 \mathrm{~A}$ \\
\hline $0,1-0,2$ & $0,8 \mathrm{~B}$ & $0,9 \mathrm{~B}$ & $1,8 \mathrm{~A}$ & $1,2 \mathrm{~B}$ & $1,8 \mathrm{~A}$ & $1,4 \mathrm{~B}$ \\
\hline \multirow[t]{2}{*}{$0,2-0,4$} & $0,8 \mathrm{~B}$ & $1,1 \mathrm{~B}$ & $1,7 \mathrm{~A}$ & $1,4 \mathrm{~A}$ & $1,8 \mathrm{~A}$ & $1,1 \mathrm{~B}$ \\
\hline & \multicolumn{6}{|c|}{$\operatorname{Mi}\left(\mathrm{m}^{3} \mathrm{~m}^{3}\right)$} \\
\hline $0-0,05$ & $4,3 \mathrm{~A}$ & $4,7 \mathrm{~A}$ & $2,9 \mathrm{~B}$ & $3,7 \mathrm{~B}$ & $3,0 \mathrm{~B}$ & $3,0 \mathrm{~B}$ \\
\hline $0,05-0,1$ & $4,2 \mathrm{~A}$ & $4,0 \mathrm{~A}$ & $2,9 \mathrm{~B}$ & $3,6 \mathrm{~B}$ & $3,2 \mathrm{~B}$ & $2,8 \mathrm{~B}$ \\
\hline $0,1-0,2$ & $4,2 \mathrm{~A}$ & $3,8 \mathrm{~A}$ & $2,9 \mathrm{~B}$ & $3,3 \mathrm{~B}$ & $2,9 \mathrm{~B}$ & $2,9 \mathrm{~B}$ \\
\hline \multirow[t]{2}{*}{$0,2-0,4$} & $4,0 \mathrm{~A}$ & $4,3 \mathrm{~A}$ & $3,0 \mathrm{~B}$ & $3,3 \mathrm{~B}$ & $2,8 \mathrm{~B}$ & $3,1 \mathrm{~B}$ \\
\hline & \multicolumn{6}{|c|}{ DMP (mm) } \\
\hline $0-0,05$ & $4,72 \mathrm{~A}$ & $4,82 \mathrm{~A}$ & $4,69 \mathrm{~A}$ & $4,70 \mathrm{~A}$ & $4,69 \mathrm{~A}$ & $4,60 \mathrm{~B}$ \\
\hline \multirow[t]{2}{*}{$0,05-0,1$} & $4,78 \mathrm{~A}$ & $4,34 \mathrm{~B}$ & $3,63 \mathrm{C}$ & $4,30 \mathrm{~B}$ & $4,33 \mathrm{~B}$ & $3,63 \mathrm{C}$ \\
\hline & \multicolumn{6}{|c|}{ DMG (mm) } \\
\hline $0-0,05$ & $4,47 \mathrm{~A}$ & $4,53 \mathrm{~A}$ & $4,26 \mathrm{~A}$ & $4,50 \mathrm{~A}$ & $4,38 \mathrm{~A}$ & $3,80 \mathrm{~B}$ \\
\hline $0,05-0,1$ & $4,34 \mathrm{~A}$ & $3,60 \mathrm{~B}$ & $2,48 \mathrm{C}$ & $3,61 \mathrm{~B}$ & $3,59 \mathrm{~B}$ & $2,61 \mathrm{C}$ \\
\hline
\end{tabular}

*Valores com a mesma letra na linha não diferem entre si pelo teste de Bonferroni a 5\%. ${ }^{1}$ FIN - Fragmento de floresta secundária em estágio inicial com 20 anos de regeneração; FIT - Fragmento de floresta secundária em estágio intermediário com 25 anos de regeneração; FAV - Fragmento de floresta secundária em estágio avançado com 60 anos de regeneração; PA - Pastagem manejada com Brachiaria decumbens e Paspalum notatum.

de regeneração das florestas secundárias (estágio inicial para avançado) (Tabela 2). Pode-se inferir que isso ocorreu devido ao maior valor de carbono orgânico total (COT) na área mais antiga de floresta secundária, a qual também apresenta um maior tempo de regeneração da vegetação, criando-se um ambiente mais favorável à presença de organismos edáficos (Menezes et al., 2009) e possibilitando a formação de bioporos (Hickmann et al., 2012).

As áreas cultivadas apresentaram valores de macroporosidade superiores às áreas de fragmento de floresta secundária em estágio inicial (FIN) e fragmento de floresta secundária em estágio intermediário (FIT) e semelhantes à área de fragmento de floresta secundária em estágio avançado (FAV) até $0,1 \mathrm{~m}$ de profundidade (Tabela 2). Após essa profundidade, apenas as áreas de pastagem (PA) (0,2-0,4 m) e agricultura perene (AP) (0,1-0,4 m) apresentaram valores superiores às áreas de FIN e FIT, e semelhantes à FAV (Tabela 2). Quanto à área de pastagem (PA), pode-se inferir que o maior valor de macroporosidade em relação às áreas de FIN e FIT pode estar relacionado ao sistema radicular das gramíneas cultivadas, que além de aumentar o conteúdo de C, favorece uma melhoria na agregação do solo. Já em relação ao maior valor de macroporosidade das áreas de agricultura em relação às áreas FIN e FIT, bem como ao valor semelhante à área $\operatorname{FAV}(0,0-0,1 \mathrm{~m})$, esse pode ser atribuído a um efeito temporário do revolvimento do solo das áreas de agricultura, pois segundo Silva et al. (2005), o revolvimento do solo promove um aumento da macroporosidade devido à ruptura dos agregados e ao aumento da proporção de espaços porosos entre os agregados.

Independentemente da profundidade analisada, as áreas de fragmento de floresta secundária em estágio inicial (FIN) e fragmento de floresta secundária em estágio intermediário (FIT) apresentaram maior valor de microporosidade em comparação às demais áreas 
estudadas (Tabela 2). Observa-se um padrão inverso dos resultados da macroporosidade, demonstrando que nas áreas FIN e FIT há uma maior concentração de microporos e, com o decorrer do tempo, regeneração, devido ao aumento do aporte de resíduos orgânicos via serapilheira e/ou sistema radicular, ocorrendo maior oferta de carbono orgânico total (COT) e consequente aumento na macroporosidade.

Já o maior valor de microporosidade das áreas de fragmento de floresta secundária em estágio inicial (FIN) e fragmento de floresta secundária em estágio intermediário (FIT) em comparação com as áreas de agricultura pode estar relacionado ao maior teor de argila e de COT, pois Silva \& Kay (1997) salientam que a microporosidade do solo é fortemente influenciada pela textura e teor de carbono orgânico e muito pouco influenciada pelo aumento da densidade do solo originada pelo tráfego de máquinas e implementos.

Na Tabela 2 pode-se verificar que, na camada superficial do solo (0,0-0,05 m), a área de agricultura anual (AA) apresentou menor valor de DMP e de DMG em comparação com as demais áreas. O padrão observado nessa área deve-se ao maior revolvimento do solo, que fraciona os agregados de maior tamanho. Resultados semelhantes foram observados por Bochner (2007), que também constatou menor DMP em uma área de agricultura em comparação com áreas de pastagem e de floresta secundária.

Na profundidade $0,05-0,1 \mathrm{~m}$ verifica-se uma redução nos valores de DMP e DMG em função do tempo de regeneração das florestas secundárias (Tabela 2). Esse resultado difere um pouco dos padrões observados na literatura, visto que com o aumento de COT em função do tempo de regeneração da floresta secundária são observados valores maiores para DMP e DMG. Esse padrão pode ter ocorrido devido à influência do sistema radicular das pastagens que antecederam a floresta. O efeito promovido anteriormente pela pastagem, em conjunto com o efeito atual das raízes da vegetação florestal, pode ter contribuído para uma maior agregação no início do processo de regeneração da floresta. No entanto, com o decorrer do tempo de regeneração da floresta, esse efeito inicial da pastagem foi minimizado, estabelecendo-se o padrão de DMP e DMG encontrado na área fragmento de floresta secundária em estágio avançado (FAV). Percebe-se então que o efeito residual da pastagem pode ter sido o diferencial para o resultado descrito anteriormente, pois, de acordo com Coutinho et al. (2010), por o sistema radicular das gramíneas ser fasciculado, proporciona maior agregação das partículas e fornecimento de carbono devido à rizodeposição.

Na profundidade 0,05-0,1 m verifica-se que a área de fragmento de floresta secundária em estágio inicial (FIN) apresentou maior valor de DMP e de DMG em comparação com as áreas cultivadas, e que, dentre essas áreas, a de agricultura anual (AA) apresentou o menor valor desse atributo (Tabela 2). O maior valor destes atributos, observados na área de FIN em comparação com as demais áreas cultivadas, deve-se ao efeito combinado da pastagem que a antecedeu em conjunto com contribuição da própria floresta, conforme explicado anteriormente. O menor valor desses atributos na área de $\mathrm{AA}$ em comparação com as demais áreas pode ser decorrente do maior revolvimento a que essa área é submetida.

Quanto à análise de componentes principais (ACP), que foi utilizada para se obter uma visão integrada dos atributos do solo para as diferentes áreas, verificou-se que para os eixos F1 e F2 as distribuições das variáveis selecionadas apresentaram variância acumulada de $84,78 \%$ para a camada $0-5 \mathrm{~cm}$ (Figura $3 \mathrm{~A}$ ) e de $87,92 \%$ para a camada 5-10 cm (Figura 3B). Esse padrão demonstra que a ACP apresenta uma margem confiável para interpretação das variáveis entre as áreas.

Para a ACP, os atributos avaliados neste estudo são representados por setas que, por sua vez, indicam a direção do gradiente máximo, em que o comprimento da seta é proporcional à correlação do atributo com os eixos e à sua importância na explicação da variância projetada em cada eixo (Gazolla et al., 2013). Verifica-se que a ACP resumiu os resultados apresentados anteriormente neste estudo, nos quais as áreas de agricultura se isolam das demais, com proximidade dos autovetores densidade do solo (Ds) e macroporosidade (Ma) e distanciamento do demais autovetores (Figura 3A, B). Esse padrão demonstra que nessas áreas o revolvimento do solo e o baixo aporte de resíduos vegetais ocasionado pela falta de adubação e calagem promovem uma redução dos teores de carbono orgânico total (COT), aumentando a densidade do solo e, no caso deste estudo, promovendo um aumento temporário da macroporosidade em função do manejo. 

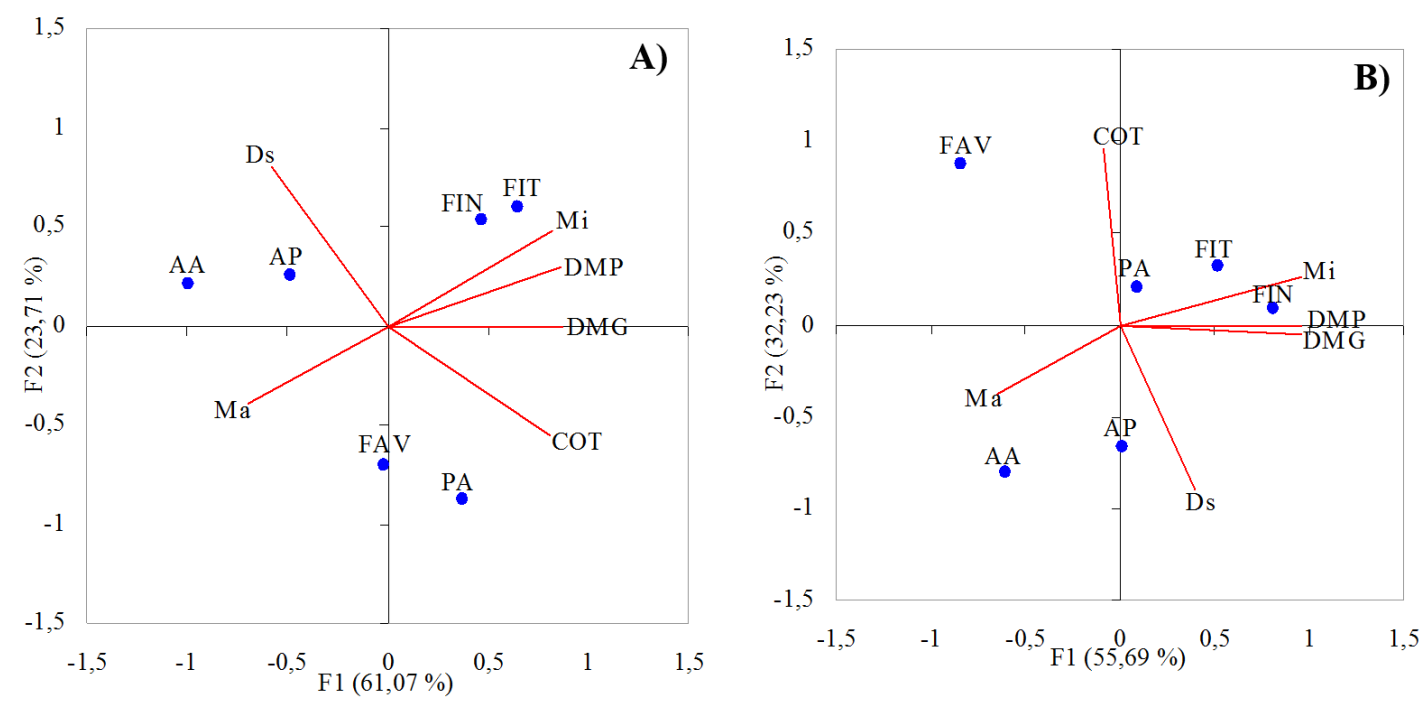

Figura 3. Diagramas de ordenação produzidos por análise de componentes principais dos atributos avaliados em amostras de terra coletadas nas camadas $0-5 \mathrm{~cm}$ (A) e $5-10 \mathrm{~cm}$ (B).

Figure 3. Ordination diagrams produced by principal component analysis, the attributes evaluated in soil samples collected layer at $0-5 \mathrm{~cm}(\mathrm{~A})$ and $5-10 \mathrm{~cm}(\mathrm{~B})$.

Percebe-se ainda a proximidade dos autovetores de COT e Ma na área de fragmento de floresta secundária em estágio avançado (FAV) e um afastamento dessa em relação às demais áreas de floresta secundária, confirmando os resultados discutidos anteriormente, nos quais o maior tempo de regeneração da FAV bem como sua maior diversidade de espécies vegetais contribuem para o aumento dos teores de COT e para a macroporosidade, reduzindo os valores de Ds.

O agrupamento dos autovetores Mi, DMP e DMG próximos das áreas de fragmento de floresta secundária em estágio inicial (FIN) e fragmento de floresta secundária em estágio intermediário (FIT) também confirma os resultados já discutidos neste estudo, nos quais a microporosidade é maior nas áreas de floresta em início de regeneração, reduzindo-se com em função do tempo de regeneração devido ao aumento da macroporosidade desencadeado pelo aumento do teor de COT e maior desenvolvimento radicular das espécies vegetais nas áreas. Já os maiores valores de DMP e DMG dessas áreas justificam-se pela contribuição do efeito residual do sistema radicular das pastagens que antecederam o processo de regeneração. Tal fato confirma-se, ainda, pela maior proximidade da área de pastagem (PA) das áreas de FIN e FIT do que com a FAV para esses atributos.

\section{CONCLUSÕES}

De maneira geral, quando comparadas as áreas manejadas, a regeneração das florestas promoveu melhorias nos atributos no solo, contribuindo para o aumento do teor de carbono orgânico e da macroporosidade, reduzindo os valores de densidade do solo e diâmetro médio ponderado e geométrico dos agregados.

Dentre as áreas estudadas, a de pastagem foi a que apresentou atributos edáficos que mais se aproximaram das áreas de floresta, sendo observado em algumas profundidades valores semelhantes de carbono orgânico total, densidade do solo, macro e microporosidade e diâmetro médio ponderado e geométrico dos agregados.

O cultivo na área de agricultura anual reduziu os teores de carbono orgânico total, prejudicando alguns atributos físicos do solo, como aumento da densidade do solo e redução de macroporosidade, diâmetro médio ponderado e geométrico dos agregados.

A agricultura perene favoreceu a manutenção dos atributos do solo: macro e microporosidade e diâmetro médio ponderado e geométrico dos agregados, que se apresentaram semelhantes aos observados nas áreas de floresta secundária. No entanto, o menor aporte de resíduos vegetais promoveu uma menor adição de carbono orgânico total e maior valor de densidade do solo. 


\section{STATUS DA SUBMISSÃO}

Recebido: 9 jun., 2014

Aceito: 19 maio, 2016

\section{AUTOR(ES) PARA CORRESPONDÊNCIA}

\section{Marcos Gervasio Pereira}

Departamento de Solos, Universidade Federal Rural do Rio de Janeiro - UFFRJ, BR-465, Km 7, CEP 23897-900, Seropédica, RJ, Brasil

e-mail: mgervasiopereira01@gmail.com

\section{REFERENNCIAS}

Balbinot R. Carbono, nitrogênio e razões isotópicas $\delta 13 C \mathrm{E}$ $\delta 15 N$ no solo e vegetação de estágios sucessionais de floresta ombrófila densa submontana [tese]. Curitiba: Engenharia Florestal, Universidade Federal do Paraná; 2009.

Barreto AC, Lima FHS, Freire MBGS, Araújo QR, Freire FJ. Características químicas e físicas de um solo sob floresta, sistema agroflorestal e pastagem no sul da bahia. Caatinga 2006; 19(4): 415-425.

Bochner JK. Serviços ambientais gerados pela floresta de mata atlântica na qualidade do solo [monografia]. Seropédica: Departamento de floresta, Universidade Federal Rural do Rio de Janeiro; 2007.

Boddey RM, Jantali CP, Macedo MO, Oliveira OC, Resende AS, Alves BJR, Urquiaga S. Potential of carbon sequestration in soils of the Atlantic Forest region of Brazil. In: Lal R, Cerri CC, Bernoux M, Etchevers J, Cerri E, editores. Carbon sequestration in soil of Latin America. New York: Howarth; 2006.

Brasil Conselho Nacional do Meio Ambiente - CONAMA. 1994. Resolução nº 6, de 4 de maio de 1994. Diário Oficial da República Federativa do Brasil, Brasília, DF (1994 maio 04) [citado em 2005 jan 25]. Disponível em: http://www. $\mathrm{mma}$.gov.br/conama/legiano1.cfm

Castro C Fo, Muzilli O, Podanoschi AL. Estabilidade dos agregados e sua relação com o teor de carbono orgânico num Latossolo Roxo Distrófico, em função de sistemas de plantio, rotações de culturas e métodos de preparo das amostras. Revista Brasileira de Ciência do Solo 1998; 22(3): 527-538. http://dx.doi.org/10.1590/ S0100-06831998000300019.

Coutinho FS. Atributos edáficos e qualidade do solo em áreas de agricultura, pastagem e fragmentos florestais na região do médio vale do paraíba do sul, Rio de janeiro [dissertação]. Seropédica: Departamento de solos, Universidade Federal Rural do Rio de Janeiro; 2012.

Coutinho RP, Urquiaga S, Boddey RM, Alves BJR, Torres AQA, Jantalia CP. Estoque de carbono e nitrogênio e emissão de $\mathrm{N}_{2} \mathrm{O}$ em diferentes usos do solo na Mata Atlântica. Pesquisa Agropecuária Brasileira 2010; 45(2): 195-203. http://dx.doi.org/10.1590/S0100-204X2010000200011.

Empresa Brasileira de Pesquisa Agropecuária - EMBRAPA. Manual de métodos de análise de solos. Rio de Janeiro: EMBRAPA; 1997.

Empresa Brasileira de Pesquisa Agropecuária - EMBRAPA. Sistema brasileiro de classificação de solos. 2. ed. Rio de Janeiro: EMBRAPA; 2006.

Fontana A, Matiello JD, Anjos LHC, Pereira MG. Fracionamento químico da matéria orgânica em solo sob diferentes coberturas vegetais em Sooretama (ES). Magistra 2005; 17(3): 135-141.

Fontana A, Silva CF, Pereira MG, Loss A, Brito RJ, Benites VM. Avaliação dos compartimentos da matéria orgânica em área de Mata Atlântica. Acta Scientiarum. Agronomy 2011; 33(3): 545-550.

Gazolla PR, Guareschi RF, Perin A. Estoque de carbono e atributos físicos de um Latossolo Vermelho em diferentes sistemas de manejo. Revista Brasileira de Ciências Agrárias 2013; 08(2): 229-235. http://dx.doi.org/10.5039/agraria. v8i2a2545.

Hickmann C, Costa LM, Schaefer CEGR, Fernandes RBA, Andrade CLT. Atributos físico-hidricos e carbono orgânico de um argissolo após 23 anos de diferentes manejos. Revista Caatinga 2012; 25(1): 128-136.

Instituto Brasileiro de Geografia e Estatística - IBGE. Manual técnico da vegetação brasileira. 2 . ed. Rio de Janeiro: IBGE; 2012.

Köppen W. Climatologia: con un estudio de los climas de la tierra. México: Fondo de Cultura Econômica; 1948. 479 p.

Loss A, Pereira MG, Brito RJ. Distribuição das substâncias húmicas em solos de tabuleiros sob diferentes coberturas vegetais. Revista Universidade Rural. Série Ciência da Vida 2006; 26(2): 57-69.

Macedo MO, Resende AS, Garcia PCM, Boddey RM, Jantalia CP, Urquiaga $\mathrm{S}$ et al. Changes in soil $\mathrm{C}$ and $\mathrm{N}$ stocks and nutrient dynamics 13 years after recovery of degraded land using leguminous nitrogen-fxing trees. Forest Ecology and Management 2008; 255(5-6): 15161524. http://dx.doi.org/10.1016/j.foreco.2007.11.007.

Machado RL, Resende AS, Campello EFC, Oliveira JA, Franco AA. Soil and nutrient losses in erosion gullies at different degrees of restoration. Revista Brasileira de Ciência do Solo 2010; 34(3): 945-954. http://dx.doi.org/10.1590/ S0100-06832010000300036.

Mateus FA, Miranda CC, Valcarcel R, Figueiredo PHA. Estoque e capacidade de retenção hídrica da serrapilheira acumulada na restauração florestal de áreas perturbadas na mata atlântica. Floresta e Ambiente 2013; 20(3): 336-343.

Melloni R, Melloni EGP, Alvarenga MIN, Vieira FBN. Avaliação da qualidade de solos sob diferentes coberturas florestais e de pastagem no sul de minas gerais. Revista 
Brasileira de Ciência do Solo 2008; 32(6): 2461-2470. http://dx.doi.org/10.1590/S0100-06832008000600023.

Menezes CEG. Integridade de Paisagem, Manejo e Atributos do Solo no Médio Vale do Paraíba do Sul, Pinheiral-RJ [tese]. Seropédica: Departamento de solos, Universidade Federal Rural do Rio de Janeiro; 2008.

Menezes CEG, Correia MEF, Pereira MG, Batista I, Rodrigues KM, Couto WH et al. Macrofauna edáfica em estágios sucessionais de floresta estacional semidecidual e pastagem mista em Pinheiral (RJ). Revista Brasileira de Ciência do Solo 2009; 33(6): 1647-1656. http://dx.doi. org/10.1590/S0100-06832009000600013.

Oliveira CM, Nappo ME, Passos RR, Mendonça AR. Comparação entre atributos físicos e químicos de solo sob floresta e pastagem. Revista Científica Eletrônica de Engenharia Florestal 2008; 7(12): 1-21.

Sá RC, Pereira MG, Fontana A. Características físicas e químicas de solos de tabuleiros em Sooretama (ES). Floresta e Ambiente 2003; 10(2): 95-99.

Santos JT, Andrade AP, Silva IF, Silva DS, Santos EM, Silva APG. Atributos físicos e químicos do solo de Áreas sob Pastejo na Micro Região do Brejo Paraibano. Ciência Rural 2010; 40(12): 2486-2492. http://dx.doi.org/10.1590/ S0103-84782010001200008.

Schedlbauer JL, Kavanagh KL. Soil carbon dynamics in a chronosequence of secondary forests in northeastern Costa Rica. Forest Ecology and Management 2008; 255(3-4): 1326-1335. http://dx.doi.org/10.1016/j.foreco.2007.10.039.
Silva AP, Kay BD. Estimating the least limiting water range of soils from properties and management. Soil Science Society of America Journal 1997; 61(3): 877-883. http:// dx.doi.org/10.2136/sssaj1997.03615995006100030023x.

Silva CF, Pereira MG, Miguel DL, Fernandes JCF, Loss A, Menezes CEG et al. Carbono orgânico total, biomassa microbiana e atividade enzimática do solo de áreas agrícolas, florestais e pastagem no médio vale do paraíba do sul (RJ). Revista Brasileira de Ciência do Solo 2012; 36(6): 1680-1689. http://dx.doi.org/10.1590/S0100-06832012000600002.

Silva GR, Silva ML Jr, Melo VS. Efeitos de diferentes usos da terra sobre as características químicas de um Latossolo amarelo do Estado do Pará. Acta Amazônica 2006; 36(2): 151-158. http://dx.doi.org/10.1590/S004459672006000200004.

Silva MAS, Mafra ÁL, Albuquerque JÁ, Bayer C, Mielniczuk J. Atributos físicos do solo relacionados ao armazenamento de água em um Argissolo Vermelho sob diferentes sistemas de preparo. Ciência Rural 2005; 35(3): 544-552. http:// dx.doi.org/10.1590/S0103-84782005000300009.

Tabarelli M, Aguiar AV, Ribeiro MC, Metzger JP, Peres CA. Prospects for biodiversity in the Atlantic Forest: lessons from aging human-modified landscapes. Biological Conservation 2009; 143(10): 2328-2340. http://dx.doi. org/10.1016/j.biocon.2010.02.005.

Yeomans JC, Bremner JM. A rapid an precise method for routine determination of organic carbon in soil. Communications in Soil Science and Plant Analysis 1988; 19(13): 1467-1476. http://dx.doi.org/10.1080/00103628809368027. 